IZA DP No. 5091

The Rise in Absenteeism:

Disentangling the Impacts of Cohort, Age and Time

Erik Biørn

Simen Gaure

Simen Markussen

Knut Røed

July 2010 


\title{
The Rise in Absenteeism: Disentangling the Impacts of Cohort, Age and Time
}

\author{
Erik Biørn \\ University of Oslo \\ Simen Gaure \\ Ragnar Frisch Centre for Economic Research \\ Simen Markussen \\ Ragnar Frisch Centre for Economic Research \\ Knut Røed \\ Ragnar Frisch Centre for Economic Research \\ and IZA
}

\author{
Discussion Paper No. 5091 \\ July 2010
}

IZA

P.O. Box 7240

53072 Bonn

Germany

Phone: +49-228-3894-0

Fax: +49-228-3894-180

E-mail: iza@iza.org

\begin{abstract}
Any opinions expressed here are those of the author(s) and not those of IZA. Research published in this series may include views on policy, but the institute itself takes no institutional policy positions.

The Institute for the Study of Labor (IZA) in Bonn is a local and virtual international research center and a place of communication between science, politics and business. IZA is an independent nonprofit organization supported by Deutsche Post Foundation. The center is associated with the University of Bonn and offers a stimulating research environment through its international network, workshops and conferences, data service, project support, research visits and doctoral program. IZA engages in (i) original and internationally competitive research in all fields of labor economics, (ii) development of policy concepts, and (iii) dissemination of research results and concepts to the interested public.
\end{abstract}

IZA Discussion Papers often represent preliminary work and are circulated to encourage discussion. Citation of such a paper should account for its provisional character. A revised version may be available directly from the author. 
IZA Discussion Paper No. 5091

July 2010

\section{ABSTRACT \\ The Rise in Absenteeism: Disentangling the Impacts of Cohort, Age and Time*}

We examine the remarkable rise in absenteeism among Norwegian employees since the early 1990's, with particular emphasis on disentangling the roles of cohort, age, and time. Based on a fixed effects model, we show that individual age-adjusted absence propensities have risen even more than aggregate absence rates from 1993 to 2005, debunking the popular hypothesis that the rise in absenteeism resulted from the inclusion of new cohorts with weaker work-norms - into the workforce. We also reject the idea that the rise in absenteeism resulted from more successful integration of workers with poor health; on the contrary, a massive rise in disability rolls during the 1990's suggest that poor-health workers have left the labor market in unprecedented numbers.

JEL Classification: $\quad$ C23, C25, I38, J22

Keywords: $\quad$ sickness absence, endogenous selection, multicollinearity, fixed effects logit

Corresponding author:

Knut Røed

Ragnar Frisch Centre for Economic Research

Gaustadalléen 21

0349 Oslo

Norway

E-mail: knut.roed@frisch.uio.no

\footnotetext{
* This paper is part of the project "Absenteeism in Norway - Causes, Consequences, and Policy Implications", financed by the Norwegian Research Council (grant \#187924).
} 


\section{Introduction}

During the last two decades, the rate at which Norwegian workers are absent from work due to sickness has risen sharply; from around 4-5 percent of paid hours in the early 1990s to around 6.5 percent today (2010). The rise in absenteeism has occurred despite general improvements in self-reported health conditions. According to Statistics Norway's level-of-living surveys, the proportion of the population reporting poor or very poor health has declined from 10 per cent in 1995 to 8 per cent in 2005 among citizens above 45 years, while it has remained stable at 3 per cent among younger citizens. Based on a sample survey on self-reported health complaints and actual absence behavior in 1996 and 2003, Ihlebaek et al. (2007) found that while the prevalence of health complaints remained stable, sickness absence rose by 65 percent.

Why has absenteeism risen so much? Generous sickness insurance - with a 100 percent replacement ratio for up to one year - can potentially explain why Norwegian absence rates are high; but without a theory of gradual adjustment of behavior in response to welfare state generosity (Lindbeck, 1995; Lindbeck et al., 1999), it cannot explain why they are rising. Today’s sickness insurance system, introduced in 1978, has undergone no improvements in coverage or generosity over the last decades. Alternative explanations abound, ranging from deteriorating work-norms or tougher workenvironments, to the inclusion of marginal (and less healthy) individuals in the workforce. The latter explanation is of particular interest in a Norwegian context, since raising marginal workers' employment propensities - together with curbing absenteeism - has been among the key goals of a tripartite "inclusive workplace agreement” between the government and the national confederations of employers and employees. If the rise in absenteeism resulted directly from the integration of marginal workers (who otherwise 
could have claimed disability benefits) policy makers would view it as a sign of success not of failure.

The purpose of the present paper is to explore the empirical relevance of the various explanations with a focus on disentangling cohort, age and time effects. Our empirical basis is individual register data on long-term absence spells (exceeding two weeks) for virtually all workers in Norway over a 13 year period (1993-2005). The data constitute a large unbalanced panel data set, with entry and exit of workers over time. While the processes generating labor market entries and exits are of substantial interest in their own right, they also represent a major problem when attempting to disentangle time, cohort and age effects in the absence pattern. A primary reason is that unobserved heterogeneity in ability and/or willingness to work is likely to be correlated both with the observed absence propensity and with entry and exit decisions. If inappropriately accounted for, this systematic sample selection will distort the estimated time, cohort and age effects. We address the sorting problem by using “fixed effects methods”, implying that we identify age and time effects on the basis of "within-worker" variation only. However, this strategy does not solve the endogenous selection problem caused by time-varying shocks affecting absenteeism and labor market participation simultaneously. Our preferred strategy for dealing with this problem is to condition inference with respect to age and time effects in absence behavior on the sample inclusion restriction that the worker is employed not only in the current, but also in the subsequent year. Since the imposition of this restriction clearly affects the interpretation of the estimated age and time effects, we examine the robustness of our findings by alternatively also including all observed worker-years in the panel data set.

The fact that individual variations in absenteeism can be ascribed, inter alia, to a combination of age and time effects brings the well-known collinearity problem caused 
by the impossibility of "time-travel" to the forefront: Since current age is the difference between current time and birth year, the two former are, for each individual, perfectly correlated. This problem arises not only when age and time are represented quantitatively, say in a linear model, but also when represented by sets of dummy variables, as we will do here. While the thought-experiment of moving individuals from one particular cohort to a time-environment experienced by another cohort is meaningful from a research perspective, such an experiment can clearly not be evaluated on the basis of observed data alone. In the present paper, we come to grips with this fundamental identification problem by imposing a minimum interpretation (identification) constraint. In essence, our basic idea is that we can identify the time (period) effects by imposing the (empirically justified) constraint that there exists a short age interval over which the age effects do not vary. Having identified the time effects, we can then easily back out the age effects, and also shed some light on differences across birth-cohorts.

Theories of social change and norm formation tend to emphasize the predominant role of entering and exiting cohorts; see, e.g., the seminal paper by Ryder (1965). A key hypothesis is that some central values are shaped by early socialization experiences in late adolescence or early adulthood and are unlikely to change in middle age and beyond (Gans and Silverstein, 2006). Lindbeck (1995, p. 11) hypothesizes that "changes in habits, norms, attitudes, and ethics are particularly likely to occur when a new generation enters working life and forms its values on the basis of a new incentive structure." Hence, if norm change were the only major force driving the increase in sickness absence, we should expect younger cohorts - who have always worked under a generous sickness insurance regime - to be stronger bearers of the new and weaker work-norm than older cohorts. Changes in job demands, on the other hand, arguably occur in a realtime dimension, thus affecting workers from all cohorts simultaneously. As a first ap- 
proximation we might thus associate cohort-effects (estimable) with norm drift (latent) and time-effects (estimable) with changes in job demands (latent). This may be overly simplistic, though, both because norms are potentially malleable at any stage of adult life and because changes in job demands may affect different cohorts differently. In other words, we cannot a priori rule out that both work-norms and job demands vary across cohorts as well as over time within cohorts.

A key finding of our paper is that individual absenteeism increased more strongly over the period 1993-2005 than what can be read off from aggregate figures. And it is the pure time-effect that dominates. While the individual absence propensity doubled between 1993 and 2003, holding age constant, for both men and women, it declined, by approximately 20 percent during 2004 and 2005. The influence of birth-cohort is evaluated with great uncertainty, since we cannot properly disentangle behavioral differences across cohorts from potential differences in their employment patterns. For example, a cohort observed in their late 50's and early 60's may tend to consist of workers with low absence rates either because the cohort is characterized by a particularly strong worknorm to start with, or because cohort-members with weaker work-norms have already been sorted out of employment. This problem is minimized by comparing cohorts in their prime-age years, since the employment rates tend to be both high and stable across cohorts (particularly for men) in this period. Focusing on cohorts that are prime aged during our data window we show that younger cohorts tend to be less absent than older cohorts, ceteris paribus. Hence, we find no evidence that work-norms have deteriorated through the entry of new cohorts.

While our results suggest that men and women have been subject to similar timeand cohort influences, we uncover substantial gender differences in the age profiles. For men, the probability of becoming long-term absent rises somewhat during the 20's and 
early 30's, is stable up to around age 50, after which it rises steeply. For women, the absence propensity rises sharply during the 20's, declines during the 30's, and then rises again from the late 40’s. The "causal” impacts of ageing on absenteeism are larger than indicated by the cross-sectional distribution of actual absence behavior across age groups. This reflects that workers with poor health are systematically sorted out of employment as they age and thus drop out of the panel under investigation.

The rest of the paper is organized as follows. Section 2 describes the institutional setting, the data, and the design of our cohort-age/time panel. Section 3 presents our empirical strategy and discusses model selection. Section 4 presents our key results, and Section 5 evaluates robustness. Section 6 concludes.

\section{Institutional setting and data}

Most Norwegian workers enjoy full coverage of lost earnings due to sickness absence for up to one year (100 percent replacement ratio). Absence spells lasting more than 3 days need to be certified by a physician (some employers admit a higher limit, up to 8 days). The first 16 days of sickness absence is paid for by the employer. After that, the bill is picked up by the Social Security Administration (SSA). Our data do not contain the absence spells paid for by the employer; only spells paid for by the SSA, i.e., those exceeding 16 days. ${ }^{1}$ The data, formally an unbalanced panel data set with the worker-year as the observation unit, cover all Norwegian employees aged 20-66 (47 different annual ages) from 1993 through 2005 (13 years), implying that employees born between 1928 and 1984 (57 different annual birth-cohorts) are included. ${ }^{2}$ A person is defined as employed

\footnotetext{
${ }^{1}$ In 2005, the sickness absence paid for by SSA accounted for approximately 75 percent of all absence days in Norway.

${ }^{2}$ We exclude persons employed directly by the state for the reason that their sickness insurance payments were not recorded by the SSA at the beginning of our data period. Note also that until April 1 1998, the employer covered only the first 14 days of the absence spell (rather than the first 16). Hence, there is a break in our data series in 1998 causing a slight decline in the level of recorded absence.
} 
in a given year if his/her annual earnings exceeded 144000 NOK (approximately 24000 USD) in that year (measured in 2009 value). A person is included in the sample in each year he/she is observed as being employed in the above mentioned sense. Sickness absence is recorded as the number of absence days paid for by the SSA for each worker in each year. Descriptive statistics for the dataset as a whole are given in Table 1, Panel I. In total, around 2.4 million workers are included in our data, contributing close to 18 million worker-years. On average, 16 percent of the men and 25 percent of the women experience a long-term absence spell each year. And conditional on having such a spell, the average number of days is 81 for men and 83 for women. Almost half of the workers have no recorded (long-term) absence spell during our observation window.

To put the development of absenteeism into perspective, Figure 1 shows the time paths of long-term absence propensities and aggregate unemployment. Long-term absenteeism trended upwards until the year 2004. It then declined sharply in response to a reform of the absence certification regulations which implied stronger activity requirements for workers with some remaining work capacity; see Markussen (2009a). Unemployment declined from 1993 to 1998, and remained at a very low level until 2002. During the first half of our observation period, it has previously been suggested that the recorded rise in absenteeism was somehow related to the tight labor market; see, e.g., Askildsen et al. (2005) and Nordberg and Røed (2009). However, from 2002 the rates of absence and unemployment have moved more or less in tandem, which casts some doubt on the hypothesis of pro-cyclicality in absence rates.

While the statistical analysis of absence behavior is based on the data summarized in Table 1, we also draw on administrative data describing the evolvement of employment and disability insurance claims for all Norwegians. These data do not enter directly into our statistical analysis, but are used to interpret our findings in Section 4 below. 
Table 1

Descriptive statistics

I. All workers
Number of persons
Number of worker-years
Mean number of worker-years per person
Percent of worker-years with long-term sickness absence
Mean number of absence days in years with absence $>0$

\section{All workers, conditional on employment next year} Number of persons

Number of worker-years

Mean number of worker-years per person

Percent of worker-years with long-term sickness absence

Mean number of absence days in years with absence $>0$

III. All workers, conditional on employment next year and with recorded long-term absence in at least one year

Number of persons

Number of worker-years

Mean number of worker-years per person

Percent of worker-years with long-term sickness absence

(a) Men

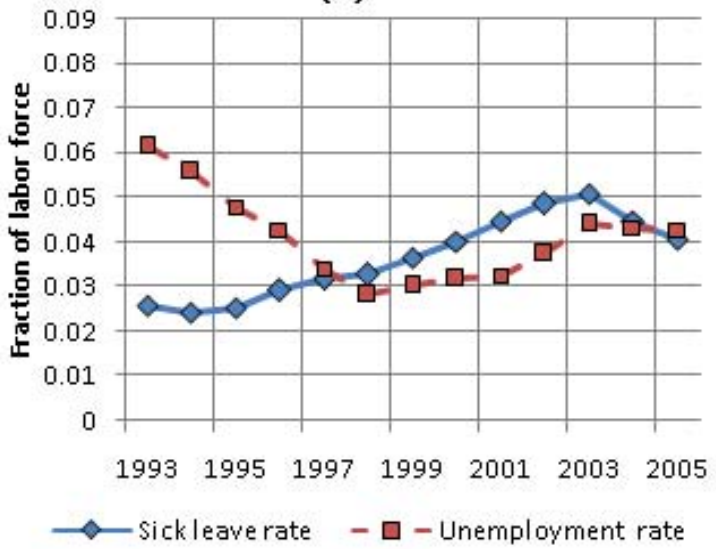

(b) Women

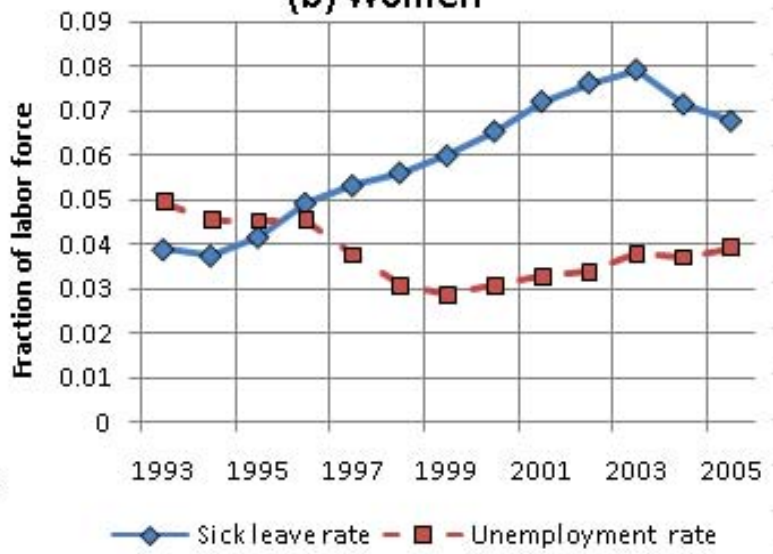

Figure 1. The rates of long-term sick leave and unemployment in Norway 1993-2005. Note: Sick leave rates are computed on the basis of the data used in the present paper (number of recorded days divided by 365). Unemployment rates are collected from the labor force sample surveys administered by Statistics Norway.

\section{Empirical analysis}

In this section we discuss and estimate models designed to separate the changes in observed absenteeism across the worker-year panel data set into birth-cohort effects, age effects, and calendar-year effects. A first question to ask is then how to measure absenteeism. Table 1 shows that the distribution of long-term sickness absence, measured in 
days, is highly skewed, with an accumulation of zeros, whereas the positive observations typically represent extensive absence. Therefore, if a fully parametric model were to be used, the choice of functional form would become a challenge; for instance, a linear regression model intended to explain the number of absence days would inadequately capture these data features. We also consider the frequency and the duration of spells to be inappropriate as dependent variables, one reason being that, e.g., a low annual spell frequency can follow from either a very low absence propensity (no absence at all) or a very high absence propensity (absent all the time). Based on these considerations, we have decided, in our basic empirical model, to explain the probability of experiencing at least one long-term absence spell during an observation year. To assess robustness, however, we also report key results when instead using linear regression with the total number of absence days in each year as the dependent variable.

\subsection{Modeling issues}

In order to identify separate cohort, age, and time effects, we face two modeling challenges. The first is the problem of endogenous sorting into and out of our dataset, which makes the panel grossly unbalanced. As time passes, new cohorts enter employment and thereby the dataset (sample accretion), while others leave, e.g., due to disability, early retirement, or death (sample attrition). These events do not occur randomly in relation to the primary research problem of the study. There is a sorting mechanism which most likely is correlated with our outcome variable, particularly because the persons who leave the sample can be expected to be more inclined to be absent - even when belonging to the same age group - than those staying. To ensure that the sorting problem do not corrupt our attempts to identify time and age effects, we apply a statistical model that controls for unobserved heterogeneity by including individual fixed effects. We recognize that individual unobserved heterogeneity in absenteeism is generated by a complex (un- 
modeled) stochastic mechanism, but we condition inference on the values of the heterogeneity variables actually realized. As a consequence, we exploit only the within-worker variation in absenteeism. Specifically, we use a binomial conditional (fixed effects) logit specification. ${ }^{3}$ Compared to alternative probability models, the logit model entails the significant practical advantage that slope coefficients can be consistently estimated without having to estimate the fixed effects (which, in our case would add up to almost 1.5 million coefficients).

The fixed effects approach is not without problems, however. Unbiased inference requires that, conditional on the fixed effects, the determination of sample inclusion (employment) logically precedes the determination of sickness absence, i.e. that the joint model of employment and absenteeism is recursive; see Biørn (2010) for an elaboration. Although the individual fixed effects can be interpreted as representing all workerspecific latent factors underlying absenteeism, such as basic health status, work-norm, occupation, work-environment, and conduct of certifying doctor, to the extent that they are approximately constant through our observation period, shocks to these variables may affect both absence propensity and (subsequent) employment. In particular, we worry that sickness absence may tend to be particularly prevalent in the last year of work-careers, not only because the risk of being absent in that year is particularly high per se (due to time and age effects), but also because long-term sickness absence - or the health shock that triggered it - may cause the work career to end. We deal with this potential problem by estimating the model without including last-year employment observations; i.e., by conditioning our worker-year observations on workers being employed in the subsequent year also. As a result, we lose approximately 10 percent of the male and 13 percent of the

\footnotetext{
${ }^{3}$ This model is described and discussed in, e.g., Chamberlain (1984, Section 3.2), Lechner et al. (2008, Section 7.3), Baltagi (2008, Section 11.1), and Hilbe (2009, Section 13.4.1)
} 
female worker-year observations; see descriptive statistics in Table 1, Panel II. While this strategy may not solve the sorting problem entirely, it provides a sound foundation for examining its empirical relevance, e.g., by comparing results from the conditional and unconditional models (where the latter includes last-year employment observations).

The second modeling problem is that our explanatory variables are perfectly collinear. By definition, current time minus birth year equals current age. Hence, if these variables occur additively in a (linear or non-linear) model, it apparently becomes impossible to identify the isolated impact of each variable separately. Technically speaking, this is due to the fact that for any individual we get only one realization of the data-generating process in the time domain. It is clearly a challenge to cope with this fundamental identification problem while seeking to recover potentially interesting "partial” age and time effects with practical applicability. Even though time-travel is impossible in the real world, it does make sense as a thought experiment, since the impacts of a purely hypothetical time-travel may convey valuable information regarding the underlying forces behind the rise in absenteeism. Our strategy for solving the multicollinearity problem is based on the imposition of an additional (minimal) restriction on the age coefficients. But before we explain that approach in more detail, we set up and estimate the more general model, where age/time effects are allowed to vary from cohort to cohort. We call this a “saturated model”, since it fully exploits the degrees of freedom in the time-age space. The drawback of this general model is that time and age effects become completely inseparable, i.e., we can only estimate a single set of time-coefficients for each cohort, which consequently must be interpreted either as age effects, as time effects or as a combination of the two. The saturated model nevertheless provides a useful benchmark against which we can evaluate (and test) the more restrictive model where age and time effects are restricted to be separable and common to all cohorts. 


\subsection{Model selection and specification}

Let $y_{i t}$ be a dichotomous outcome measure equal to 1 if worker $i$ had at least one longterm absence spell in year $t$, and zero otherwise. The imposed logit form of the probability implies that the log-odds of absence versus non-absence can be written as:

$$
\begin{aligned}
& \ln \frac{\operatorname{Pr}\left[y_{i t}=1 \mid \alpha_{i}, t\right]}{\operatorname{Pr}\left[y_{i t}=0 \mid \alpha_{i}, t\right]}=\alpha_{i}+\beta_{c t}, \\
& i=1, \ldots, N ; c=1928, \ldots, 1984 ; t=1993, \ldots, 2005
\end{aligned}
$$

where $\alpha_{i}$ is a worker-specific fixed effect, and $\beta_{c t}$ is the impact of being in year $t$ for members of cohort $c$. Note that this setup implies that each year is associated with a separate coefficient for each cohort $\left(\beta_{c t}\right)$. Apart from the imposed logit form with individual and age/time effects entering additively, there is no functional form restriction on the way age/time is assumed to affect absence propensity. There is, on the other hand, an important restriction embedded in the assumption that the time profiles entering the logit exponentials are the same for all workers belonging to the same cohort. Worker-specific age/time coefficients ( $\beta_{c t}$ replaced by $\beta_{i t}$ ) would have been infeasible, as they cannot be uncovered from data with only one time series existing for each individual. Note, however, that the logit structure implies that individual effects and cohort effects actually interact in the sick-leave probability, though they are separable in the corresponding log-odds ratio; see, e.g., Greene (2008, Section 23.11.1).

We estimate the cohort-specific age/time effects $\left(\beta_{c t}\right)$ by means of fixed effects logit model with no less than 541 age/time dummy variables. Due to the inclusion of individual fixed effects, it is only workers with variation in the outcome variable that can be used to identify the parameters of interest. Since around 54 percent of the men and 43 percent of the women in our data set never experience long-term absence during our observation window, the "effective dataset" is reduced to around 1.1 million individuals 
with 10 million worker-years; see Table 1, Panel III for details. Figure 2 illustrates the estimation results by taking a closer look at the absence behavior of four of the 57 cohorts, namely those born in 1940, 1950, 1960, and 1970. These cohorts have in common that they are observed for all the 13 years in our data-window, but of course at different ages. In Figure 2, we plot the estimated cohort-specific age/time absence profiles together with the observed absence rate by year for these four cohorts. Observed absence rates are plotted for the effective dataset (workers with variation in the outcome variable) as well as for all workers (including those with no absence in the observation window). The point estimates are normalized such that all profiles equal observed absence frequencies in the effective dataset in 1993. The graphs illustrate two important points. First, the rate of long-term absence has increased significantly over time for members of all the four cohorts (apart from a decline the last two calendar years), and the calendar-time patterns in the estimated age/time effects are conspicuously similar across cohorts. For the prime aged (represented in the graphs by the 1950, 1960, and the 1970 cohorts), in particular, the age/time effects exhibit a similar time-pattern. This suggests that when pooling different cohorts in the data set, a sensible working hypothesis, potentially useful as an identification restriction, may be that time and age effects are cohort-invariant. The second point to note by comparing the three graphs for each cohort in Figure 2 is that there are large differences between the changes in observed absence behavior within cohorts (as represented by the data), on the one hand, and the corresponding changes when the individual heterogeneiety has been controlled for, i.e., within individuals (as represented by the estimates), on the other. This suggests that sorting into and out of employment is of paramount importance for understanding a cohort's recorded aggregate absence over time. Unsurprisingly, the sorting process is much more prevalent for older than for 
younger cohorts: workers with high individual absence propensity are sorted out of employment as they age, and this sorting process intensifies at higher ages.

(a) Men

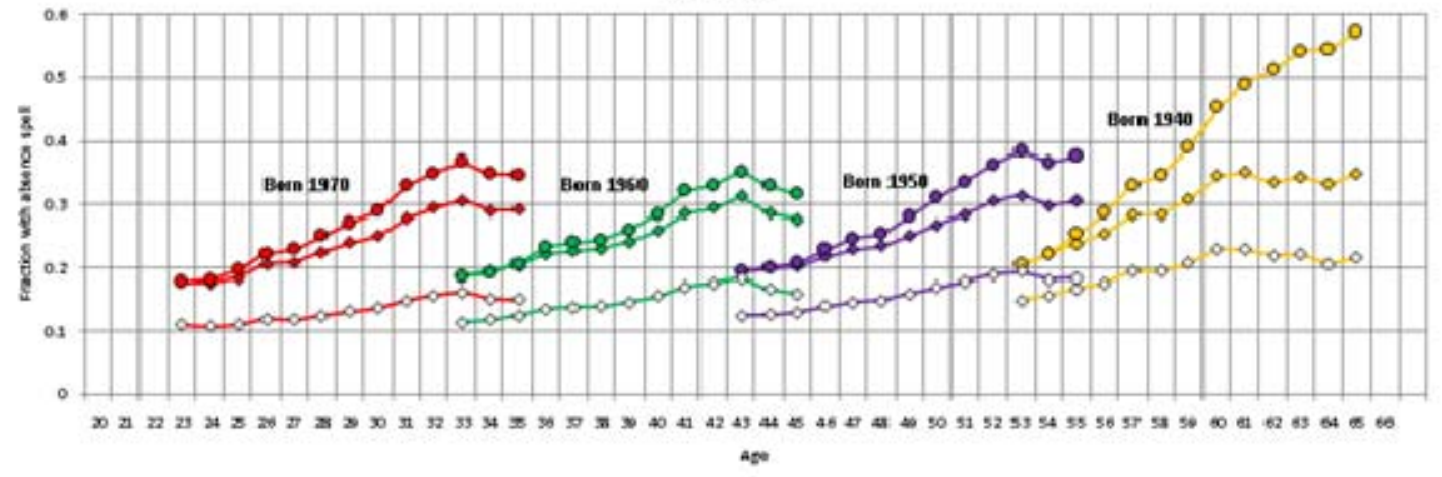

(b) Women

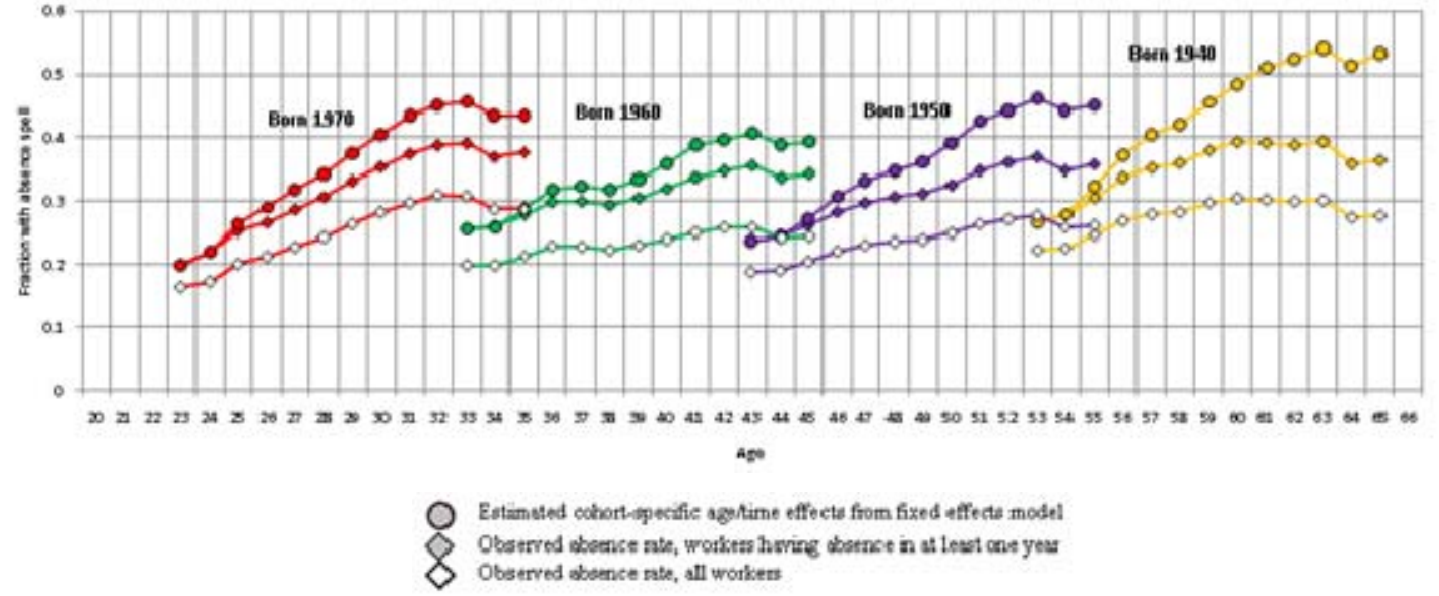

Figure 2. Estimated cohort-specific age/time effects from fixed effects model and observed absence rates by age and cohort. Selected cohorts only.

Note: Estimated age/time effects are normalized by scaling the intercept in Equation (1) such that they are equal to actual absence rates in the (effective) analysis population 1993 (the first observation year for all the cohorts in the graph)

We now turn to a logit model with time and age coefficients a priori restricted to be cohort invariant, by exploiting the working hypothesis referred to above. By restricting the model's time/age pattern in this way, we can circumvent the identification problem inherent in Equation (1) that time effects perfectly mirror age effects and vice versa. We retain, however, the worker-specific. The logit model then implies a log-odds absence versus non-absence ratio of the form

$$
\ln \frac{\operatorname{Pr}\left[y_{i t}=1 \mid \alpha_{i}, t, a_{i t}\right]}{\operatorname{Pr}\left[y_{i t}=0 \mid \alpha_{i}, t, a_{i t}\right]}=\alpha_{i}+\beta_{t}+\beta_{a} a_{i t},
$$


where $a_{i t}$ is a vector of age dummies (one for each yearly age). Since time and age is perfectly correlated at the individual level, we have a within-group collinearity in Equation (2). ${ }^{4}$ Hence, even after imposing a zero restriction on a reference age and on a reference calendar year, the parameters $\left(\beta_{t}, \beta_{a}\right)$ cannot be readily identified. From Theorem 3.2 in Kupper et al. (1983) it follows that what can actually be identified in Equation (2) without further restrictions are the differences between pairs of coefficients with the same distance; see Gaure (2010) for details. To illustrate, let $\beta_{a, r}$ denote the age coefficient associated with age $r$. We can then readily identify the differences between any two differences with distance 1: $\left(\beta_{a, s+1}-\beta_{a, s}\right)-\left(\beta_{a, r+1}-\beta_{a, r}\right)$. However, in order to arrive at interpretable marginal effects, we need to determine the value of a reference difference, in addition to selecting the trivial reference age. Since this reference acts as a normalization not a restriction - it is in some sense immaterial. However, the interpretation of each coefficient set estimated in this way will depend heavily on which specific ages are selected and the value assigned to the coefficient difference. Let $a=r$ be the immaterial reference age $\left(\beta_{a, r}=0\right)$, and let $\left(\beta_{a, r+1}-\beta_{a, r}\right)=\beta_{a, r+1}$ be the chosen reference difference. Since every difference of distance 1 is identified, arbitrary differences from our referece age are also identified by the identity

$$
\beta_{a, r+S}-\beta_{a, r}=\sum_{t=r}^{r+S-1}\left[\left(\beta_{a, t+1}-\beta_{a, t}\right)-\left(\beta_{a, r+1}-\beta_{a, r}\right)\right]+S\left(\beta_{a, r+1}-\beta_{a, r}\right)
$$

The sum to the right is the partial effect of increasing age by one year from $t$ to $t+1$, relative to increasing it from $r$ to $r+1$. As all the terms on the right hand side are identified by the above normalization, the left hand side is also identified. It is then clear that if we

\footnotetext{
${ }^{4}$ Let $\left(t_{r}, a_{r}\right)$ be the reference year and reference age, respectively, with $c_{r}=t_{r}-a_{r}$. Take a person in cohort $c$ at time $t$ and age $a$ (so tat $t-a=c$ ) with time dummies $t_{j}\left(t_{j}=1\right.$ for $j=t$ ) and $a_{j}\left(a_{j}=1\right.$ for $\left.j=a\right)$. We then have that $\sum_{j}\left(j-t_{r}\right) t_{j}-\sum_{j}\left(j-a_{r}\right) a_{j}=t-t_{r}-\left(a-a_{r}\right)=c-c_{r}$, which is obviously constant within each individual.
} 
knew a priori that there were no difference in age effects between $r$ and $r+1$, we have that $\beta_{a, r+S}$ can be interpreted directly as the effect of changing age from $r$ (or from $r+1$ ) to $r+S$, holding calendar time constant. If, on the other hand the assumption that $\beta_{a, r+1}=\beta_{a, r}$ is incorrect, the interpretation error we make is proportional to the length of the hypothetical age-travel $S$. The same considerations hold true for the time coefficients, but the error is still proportional to the error we make in the interpretation of the age coefficients. That is, the error we make when changing time by the amount $S$ (without changing age) will be $S\left(\beta_{a, r+1}-\beta_{a, r}\right)$. Thus, the error in the a priori assumption $\beta_{a, r+1}=\beta_{a, r}$ propagates to the time coefficients, and is multiplied by the distance from their reference.

We are indeed going to impose a normalization restriction corresponding to a difference that we will claim is equal to zero. Note, however, that the model's statistical validity - in terms of its ability to fit the data and make predictions - is not at all dependent on the validity of this assumption. The restriction lies in the interpretation of the distinct coefficient sets. Hence, its correctness will be crucial for our ability to assess the effects of hypothetical time- or age traveling, but will have no impact whatsoever on our ability to assess the overall effects of feasible movements across time and age (since feasible movements always involve a change in both time and age). The reason why a valid restriction on a difference between two coefficients permits us to travel in time is highly intuitive; if we know a priori that, say, $\beta_{a, r+1}=\beta_{a, r}$, then it is obviously the case that the change in absenteeism occurring as persons of age $r$ become $r+1$ years identifies the pure time effect of moving between the corresponding two calendar years. Having identified the time effects for all years, it is then trivial to identify the remaining age effects.

The normalization we have chosen to apply in the current application builds on the observation that there is a short age-span for which previous evidence indicates a constant age effect; see, e.g., Markussen et al. (2009). In particular, there is a short period 
around the age of 40 where the absence behavior does not seem to change at all and where employment rates are also virtually constant. In our own data, we indeed find that within-calendar-year differences in absence rates across all neighboring ages between 35 and 45 are small. Based upon a closer inspection of these differences, we have selected the assumptions that there are no differences between age 37 and age 38 for men, and no difference between age 42 and 43 for women. ${ }^{5}$ To examine the robustness of the resultant coefficient interpretations, we also present interpretations based on alternative normalizations. As it turns out, the estimated calendar time and age effects are highly robust towards reasonable variations in the identifying assumptions.

Table 2

Performance of the models with cohort-specific (unrestricted) and cohort-invariant (restricted) coefficients

\begin{tabular}{l|cc|cc}
\hline & \multicolumn{2}{|c|}{ Men } & \multicolumn{2}{c}{ Women } \\
\hline Number of estimated parameters & Unrestricted & Restricted & Unrestricted & Restricted \\
Number of observations (persons) & 541 & 57 & 541 & 57 \\
Information criteria & 668587 & 668587 & 759251 & 759251 \\
Log-likelihood & & & & -2134534 \\
CAIC & -1985570 & -1986161 & -2133503 & 4269897 \\
BIC & 3978937 & 3973144 & 4274872 & 4269840 \\
Prediction properties & 3978396 & 3973087 & 4274331 & 69.10 \\
Percent correct year predictions & & & & 17.25 \\
Percent correct prediction sequences & 71.70 & 72.41 & 67.72 & 0.02207 \\
Goodness of fit & 15.30 & 16.43 & 14.48 & 0.2027 \\
McFadden R-square & & & & 0.02254 \\
Prediction distance measure $(\rho)$ & 0.02318 & 0.02289 & 0.1262 & \\
\hline
\end{tabular}

Note: $\mathrm{CAIC}=-2 \ln L+(1+\ln (\mathrm{N})) \mathrm{q}$ BIC=-2lnL+ln(N)q.; see Cameron and Trivedi (2005, p 279). The Prediction distance measure $(\rho)$ is explained in the text and (more thoroughly) in a separate Appendix.

Before we present the result from the model with cohort-invariant time and age effects, we evaluate its empirical validity against the saturated model; see Table 2 . Note first that the restricted model is rejected on pure statistical grounds (for "common” pvalues) - for both men and women. The likelihood ratio test statistics are 1182 and 2062, respectively, which can be measured against the relevant five percent rejection threshold (with 484 degrees of freedom) of 536. However, given our large dataset, we would ex-

\footnotetext{
${ }^{5}$ The average within-year difference in the absence rate between men aged 37 and 38 and women aged 42 and 43 (1993-2005) are both around 0.01 percentage points.
} 
pect most model restrictions to be rejected, even when they are close approximations to the true data generating process. The question we want to answer in our case is not really whether the assumptions of common age and time effects are strictly true, but whether they represent a sufficiently good approximation to the reality to be of help in our attempts to explain changes in absenteeism over time. The various performance measures reported in Table 2 indeed indicate that the restricted model performs well. According to the two information criteria reported (CAIC and BIC), the restricted model clearly outperforms the saturated model for both men and women.

We also report the two models' prediction properties. Since the fixed effects $\left(\alpha_{i}\right)$ are not identified, we are not able to make an ordinary prediction for each individual. However, within an individual, the order of the probabilities is identified. If a person has $M$ observed positive outcomes, the obvious prediction based on our model is that the $M$ years with the highest probabilities of positive outcomes are observed with this result. The number reported in Table 2 as "percent correct year predictions" is the percentage of years with correctly predicted outcome, while the number recorded as "percent correct prediction sequences" is the percentage of workers with all years correctly predicted. It is clear from the results reported in Table 2 that the restricted model has higher score according to this criterion than the more general model. We also use two measures of goodness of fit. The first is McFadden's R-square, which is slightly higher for the saturated model, reflecting its larger likelihood. The second $(\rho)$ is a prediction distance measure. For each person we measure the distance between the predicted sequence and the worst possible prediction. We then compute the normalized Kendall distance, the number of adjacent exchange operations needed to transform one vector into another, and normalize by dividing by the distance between the best and worst prediction. Finally, we rescale such that $\rho$ is zero for random data, 1 for the perfect prediction and -1 for the 
worst possible prediction; see the Appendix for details. Again, we find that the restricted model outperforms the more general model.

(a) Men

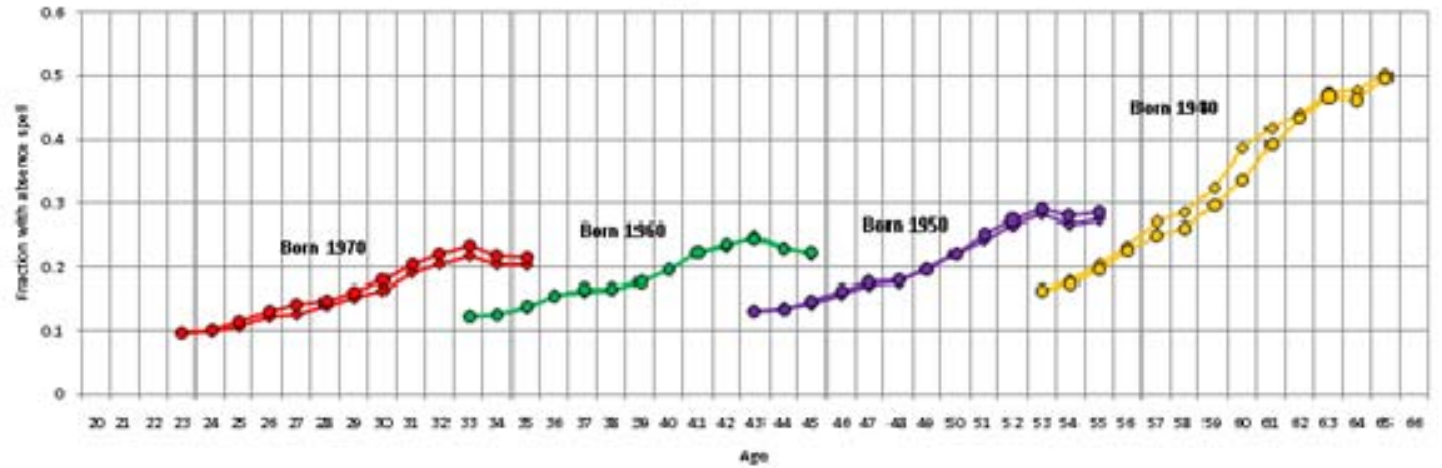

(b) Women

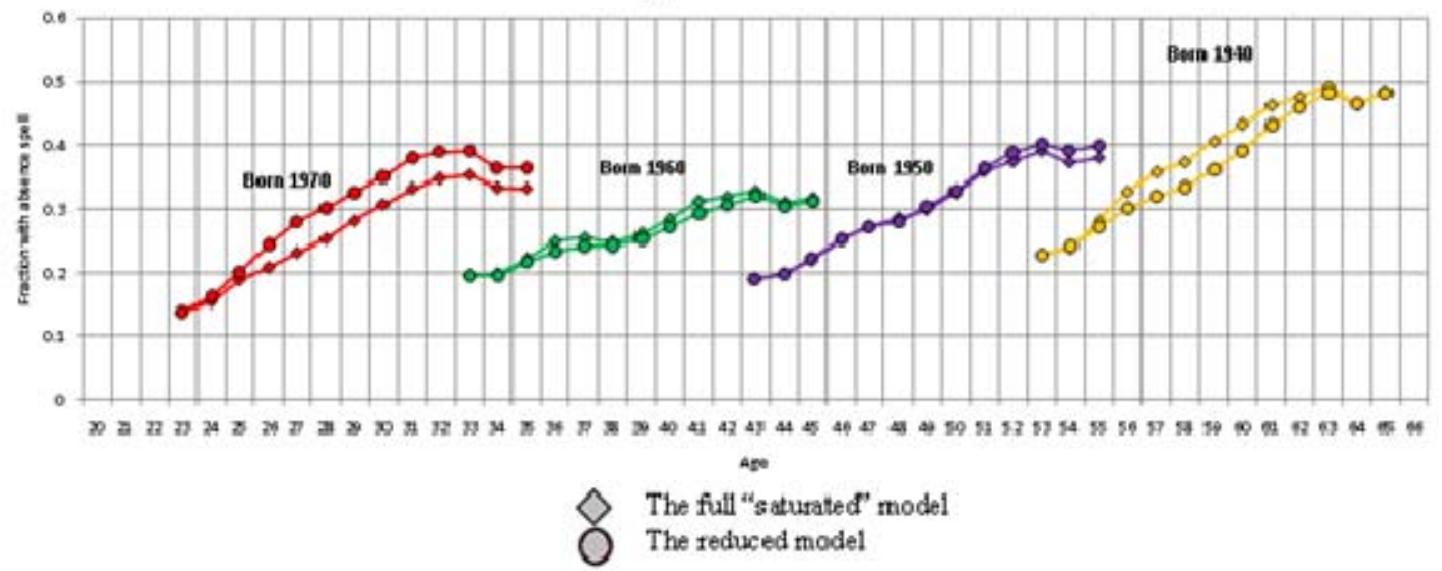

Figure 3. Estimated cohort-specific age/time effects from saturated model and model with cohort-invariant time and age coefficients. Selected cohorts only.

Note: Estimated age/time effects are normalized on actual absence rate in 1993 (the first observation year for all the cohorts in the graph)

Another way of assessing the performance of the restricted model is to compare its cohort-specific age/time profiles with those of the more general model. In Figure 3, this is done for the same four cohorts that we presented in Figure 2. For men, the restricted model appears to perform extremely well. For women, we note a tendency for the restricted model to over-estimate the rise in absenteeism for younger cohorts, while it under-estimates it for older cohorts. We nevertheless conclude from these exercises that the model with cohort-invariant time and age coefficients offers an appropriate tool for a more detailed examination of the mechanisms behind changes in absenteeism over time. 


\section{Estimation results and implied cohort differences}

Estimation results for the restricted model (Equation 2) are presented with 95 percent confidence intervals. Figure 4 presents the estimated age effects for men and women, respectively, together with the series of observed absence rates by age. Observed absence rates are again plotted for the analysis population actually used to identify the parameters of interest as well as for all workers. The point estimates are scaled such that the predicted absence rate exactly matches the observed absence rate in the (effective) analysis population at the reference age (37-38 for men, $42-43$ for women). It is evident that the within-individual probability of becoming long-term absent rises much more strongly with age than the between-individual probability (here represented by the data). The explanation is that early labor market entrants - i.e., those who drop out from school early are negatively selected and have higher absence propensities than later entrants. For men, we see a similar pattern at higher ages. The explanation for this phenomenon is that workers with high absence propensity are systematically sorted out of the labor market as they age. It may be noted from Figure 4 that there are some substantial differences in absence patterns between men and women, both with respect to the overall level and the age-profile. Except at very young and very old ages, women tend to be much more absent than men. And unsurprisingly, the difference is particularly marked during the reproductive phase of women's lives. While it is natural to interpret the causal age gradient at higher ages as reflecting deteriorating health, it is perhaps more difficult to understand the sharp rise in absenteeism at early ages, particularly for men. We speculate that it is related to tenure, job security, and learning; i.e., that workers make every effort to avoid unnecessary sickness absence during the initial stages of employment where the cost of absenteeism is particularly high - in terms of increased layoff risk and poorer career prospects; see Markussen (2009b). 
Men

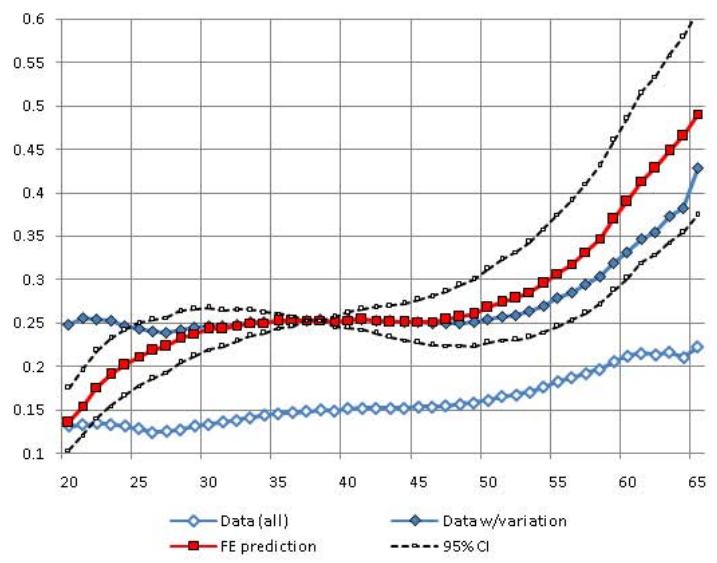

Women

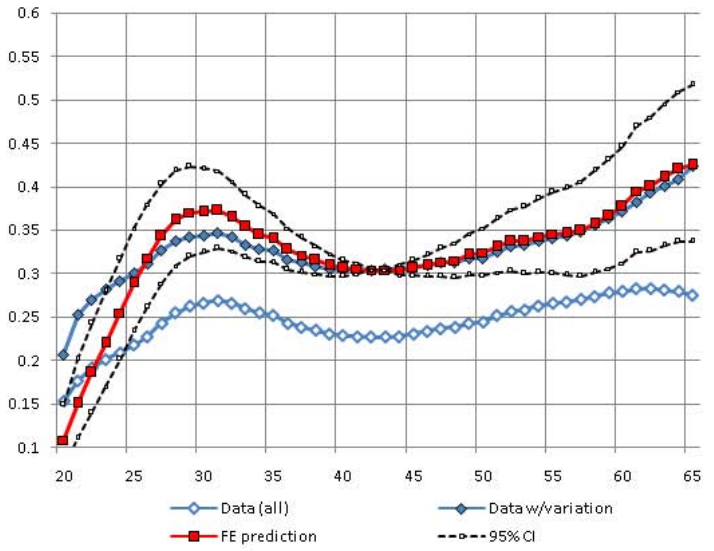

Figure 4. The estimated impacts of age on the probability of experiencing a long-termsickness episode during a one-year period.

Note: The graphs are normalized such that they match the observed absence frequency at age 37 for men and at age 43 for women. The two data series report the average annual absence rates by age taken over all observations in the dataset (excluding last year observations) and over observations belonging to workers with variation in the outcome variable, respectively.

Men

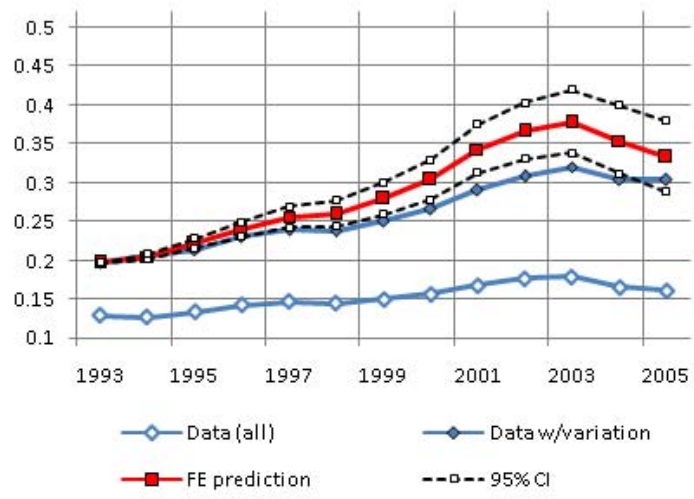

Women

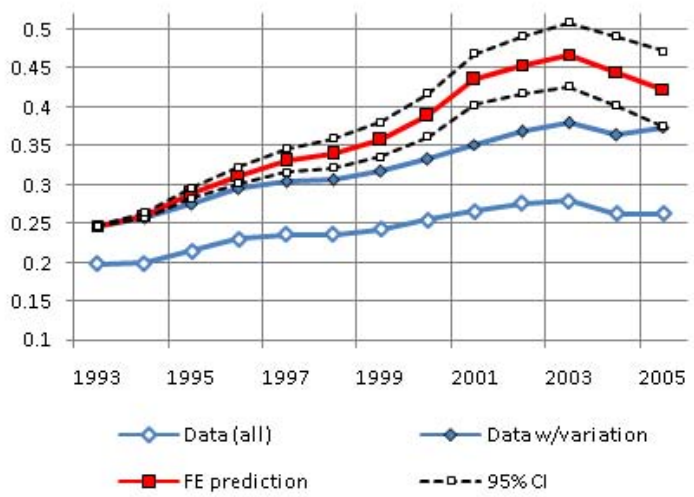

Figure 5. The estimated impacts of time (calendar year) on the probability of experiencing a long-term-sickness episode.

Note: The graphs are normalized such that they match the observed absence frequency in 1993, for men and women, respectively.

Figure 5 presents the estimated calendar time (period) effects, together with observed aggregate absence frequencies by calendar year. The estimated effects are scaled to match the observed absence rates in 1993. The key message coming out of Figure 5 is that individual absence rates have risen more over time (conditional on age) than actual absence rates. This finding suggests that the sorting process into and out of employment has actually contributed to a decline in sickness absence; i.e., that individuals with high 
individual absence propensity have been disproportionably pulled or pushed out of employment.

Our modeling framework does not offer any simple way of assessing the changes in absence propensity across cohorts (conditional on time and age), since a cohort's absence behavior during a limited time window is bound to reflect both the individual cohort-members intrinsic absence propensity and the sorting process determining which of the cohort members that are employed within the selected time window (the distribution of individual fixed effects). A cohort's age-time-adjusted absence behavior may nevertheless convey useful information. Let $\bar{y}_{c t}$ be the observed mean cohort absence rate in a particular year (including workers with only zero-absence observations); i.e., $\bar{y}_{c y}=\frac{1}{N_{c t}} \sum_{i \in c} y_{i t}$, where $N_{c t}$ is the number of employed workers from cohort $c$ in year $t$. We compute a cohort-time-parameter $\alpha_{c t}$ by solving the equation

$$
\bar{y}_{c t}=\frac{\exp \left(\alpha_{c t}+\hat{\beta}_{t}+\hat{\beta}_{t-c}\right)}{1+\exp \left(\alpha_{c t}+\hat{\beta}_{t}+\hat{\beta}_{t-c}\right)} \Rightarrow \alpha_{c t}=\ln \left(\frac{\bar{y}_{c t}}{1-\bar{y}_{c t}}\right)-\hat{\beta}_{t}-\hat{\beta}_{t-c}
$$

We then normalize by computing

$$
\alpha_{c t}^{*}=\frac{\exp \left(\alpha_{c t}\right)}{1+\exp \left(\alpha_{c t}\right)}
$$

which summarizes the employed members of cohort $c$ 's absence propensity in year $t$, adjusted for age and time effects. The two upper panels of Figure 6 shows the estimated age-time adjusted absence propensities in $2005\left(\alpha_{c 2005}^{*}\right)$ by birth cohort for men and women, respectively. The confidence intervals are computed on the basis of the nonparametric bootstrap; i.e., we have re-sampled (with replacement) and re-estimated the model 120 times - each time computing new vectors of absence propensities according to Equations (3) and (4) - and then finally excluded the three most extreme results at each 
MEN, 2005

(a) Age-time adjusted absence propensity

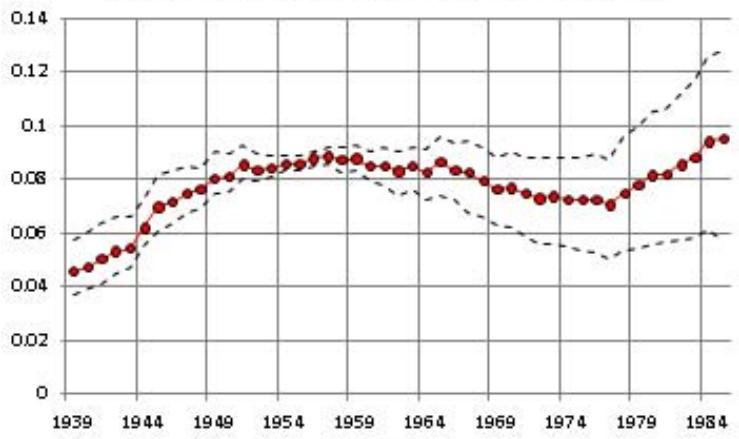

(c) Temporary and permanent disability

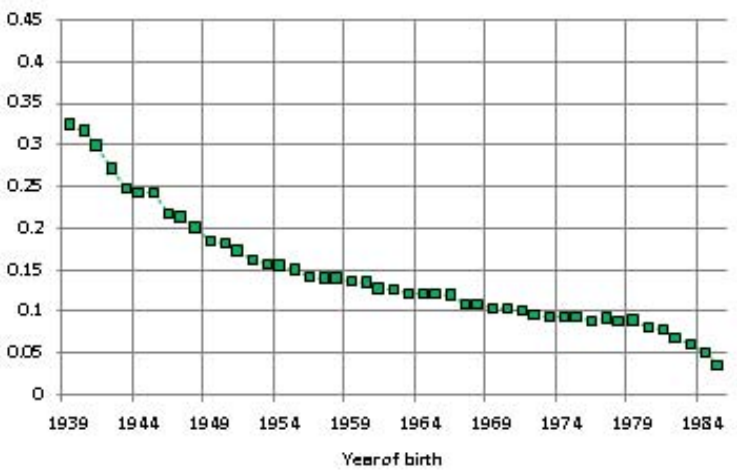

(e) Employment rate

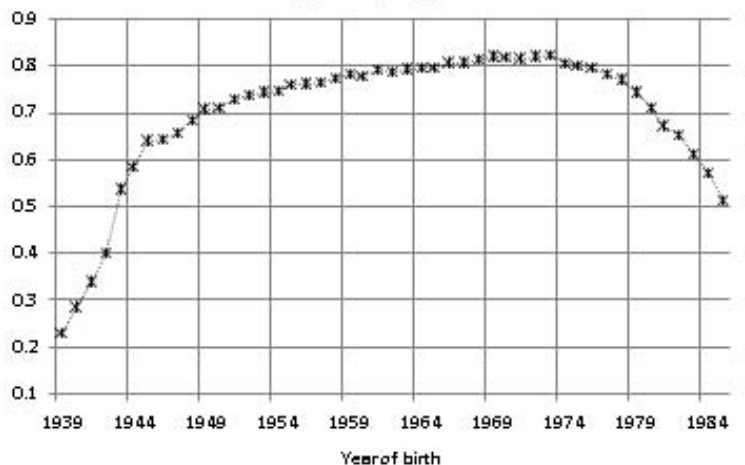

WOMEN, 2005

(b) Age-time adjusted absence propensity

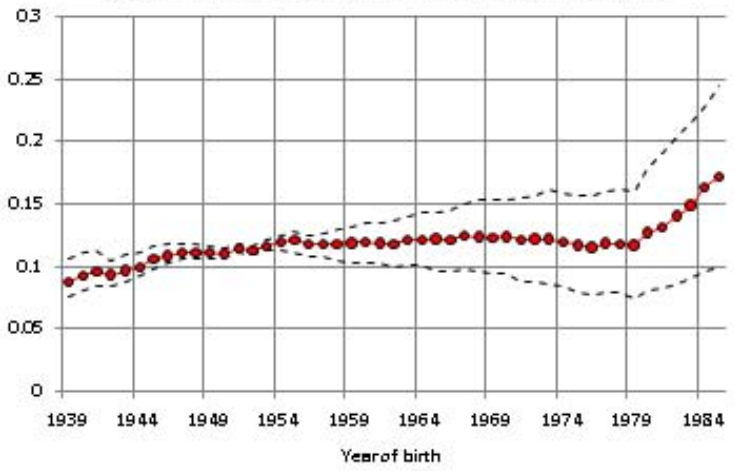

(d) Temporary and permanent disability

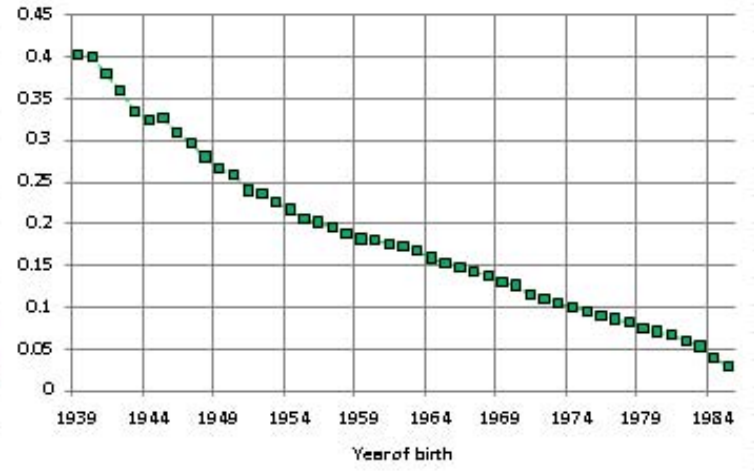

(f) Employment rate

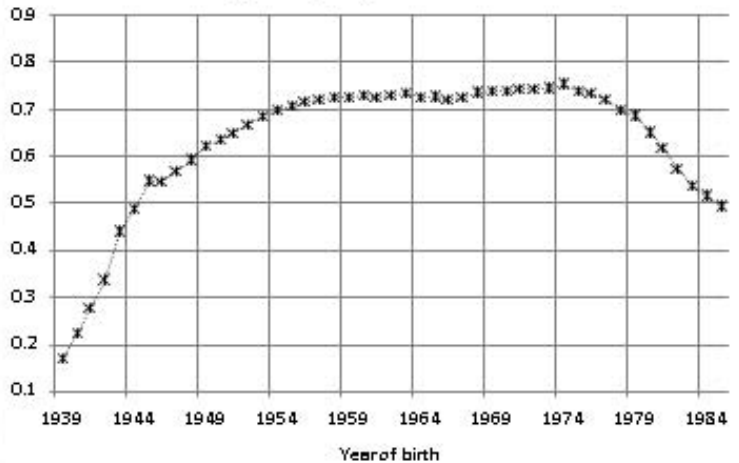

Figure 6. Estimated age-time-adjusted absence propensity (panels a) and b)), rates of temporary and permanent disability (panels c) and d)), and rates of employment (panels e) and f)). All numbers by year of birth in 2005 .

Note: Temporary and permanent disability includes disability pension and rehabilitation benefits (in relation to medical and/or vocational rehabilitation)

end for each $\alpha_{c t}^{*}$. It is clear that older workers on average have lower (age-time adjusted) absence propensities than younger workers. This pattern is likely to reflect sorting. To illustrate some potentially important sorting processes, we report in the lower four panels of Figure 6 the cohorts' rates of disability benefit dependency (panels c) and d)) and em- 
ployment (panels e) and f)) in 2005. The average absence propensity declines as a cohort's employment rate rises during the years of school-to-work transitions (moving from the extreme right in the graphs). This may suggest that workers who enter the labor market in their early 20's are adversely selected (in terms of their absence propensity). It declines further when the cohort's employment rate falls during the years of rising disability benefit dependency (moving towards the extreme left in the graphs). It seems probable that workers who are still employed in their late 50's and early 60’s (born before 1955) are favorably selected.

Given the systematic sorting out of the labor force and thus out of our analysis population, it is perhaps surprising that the absence propensity among the remaining workers do not decline more when we move towards the left in Figure 6, from younger to older cohorts. One possible explanation is simply that younger cohorts are less absent than older cohorts, ceteris paribus, e.g. because of health improvements or because worknorms of younger cohorts have improved relative to older cohorts. In order to address the issue of changes in behavior across cohorts, however, we really need to compare cohorts at similar employment levels. Figure 7 provides a comparison of the 1953-1965 birth cohorts at age 40 (the other cohorts are not observed at age 40 in our data window), at which point employment rates tend to peak within cohorts. For both men and women, we find a significant decline in the cohorts’ absence propensity; see panels a) and b). Other things equal, a member of the 1965-cohort is approximately 30 percent less likely to have a long-term absence spell during a year than a member of the 1953-cohort. This finding does apparently not support the idea that younger cohorts subscribe to a weaker worknorm than older cohorts. However, Figure 7 also shows that there has been a tremendous rise in disability rates at age 40 over these 13 birth-cohorts (panels b) and c)), and from the 1960-cohort and onwards, there has also been a slight decline in employment rates. 
Hence, an employed 40 year old person from the 1965-cohort is likely to be more favorably selected than his/her 1953 counterpart. Without a proper model for employment, we are not able to disentangle the forces of sorting from genuine differences across cohorts. What we can say, however (based on Figure 5 above) is that individual absenteeism has risen significantly more than aggregate absenteeism; hence, if the rise in absenteeism is somehow caused by norm deterioration it seems clear that the formation of a new and weaker work-norm is not particularly driven by the entry of new cohorts.

MEN, AGE 40

(a) Absence propensity

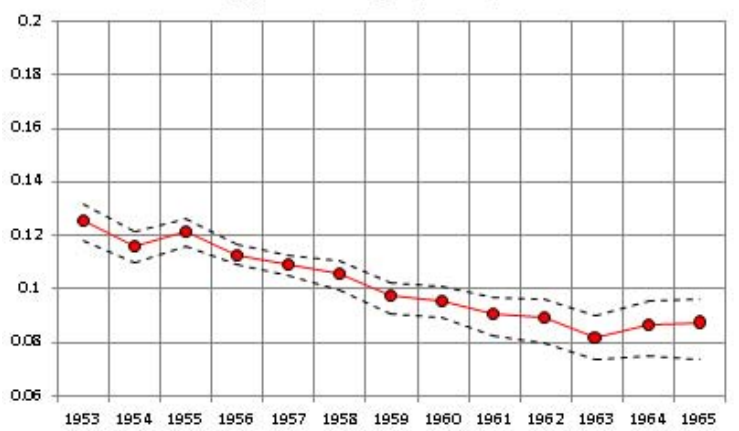

(c) Temporary and permanent disability

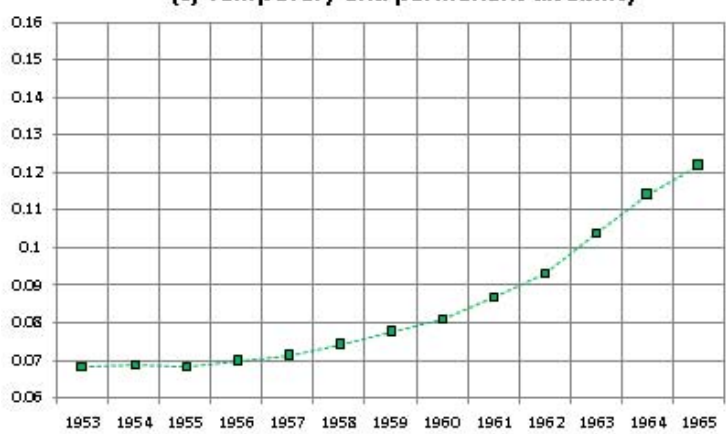

(e) Employment rate

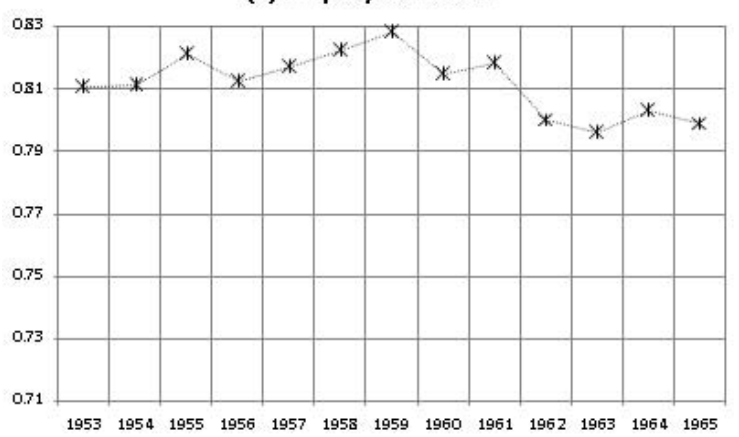

WOMEN, AGE 40

(b) Absence propensity

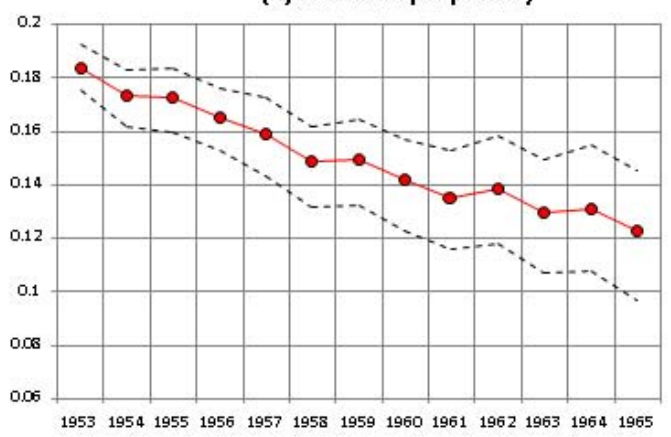

(d) Temporary and permanent disability

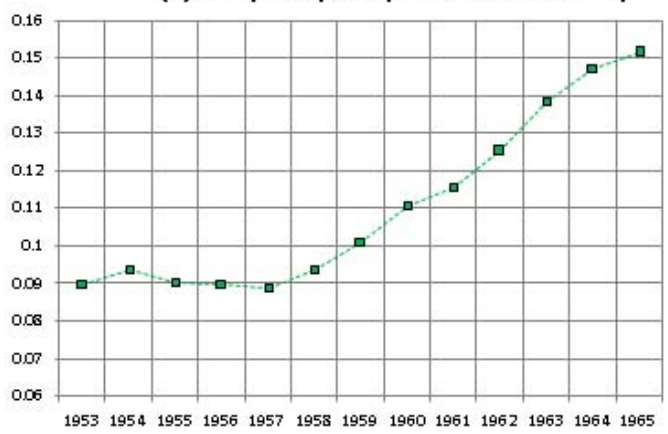

(f) Employment rate

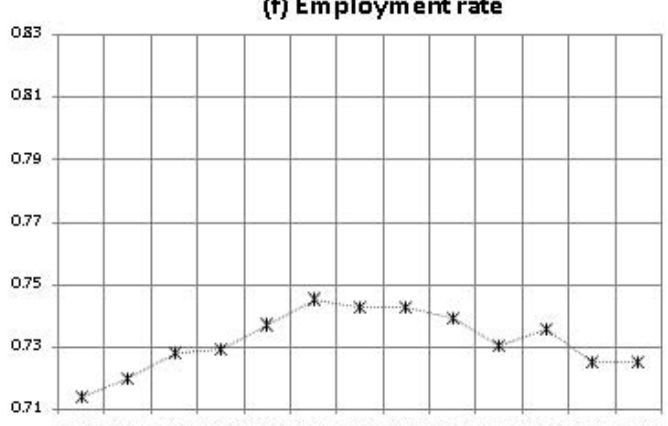

Figure 7. Estimated age-time-adjusted absence propensity by cohort, conditional on employment at age 40 . 


\section{Robustness}

In this section, we examine the robustness of our results with respect to i) the inclusion of individuals' last employment year in the estimation, ii) the selection of interpretation restriction facilitating travels in time and age, and iii) the selection of outcome measure and functional form. We focus on the robustness of the calendar year coefficients in this section, given that these are the coefficients of greatest interest and noting that the degree of robustness tends to be similar for the time and age coefficients. Note, however, that since interpretation errors are proportional to the length of the time- or age travel, our age profiles are less robust towards interpretation errors than the time profiles for the simple reason that they involve much longer journeys (while the estimated time-profile covers 13 years, the age profile covers 47 years).
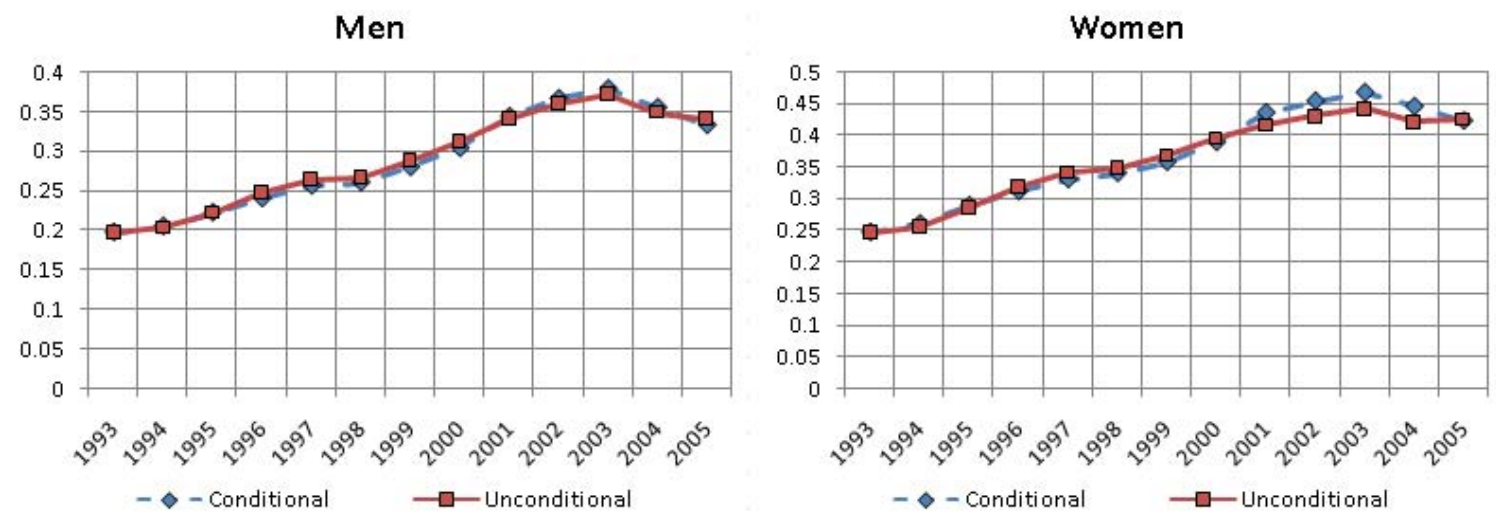

Figure 8. The estimated impacts of time on the probability of experiencing a long-tersickness episode with (unconditional) and without (conditional) last-year observations included.

Note: The series are normalized to be equal in 1993 (to the observed absence frequencies in the conditional dataset).

Figure 8 displays the estimated calendar time effects from a model based on data that comprise workers’ final-year employment observations (unconditional model) together with the previous estimates (conditional model). If endogenous sorting due to health shocks that simultaneously affect absenteeism and employment is empirically im- 
portant, we would expect the inclusion of final-year observations to change results significantly. As it turns out, it does not. For men, the two series are hardly distinguishable.

We now turn to the issue of robustness with respect to the identifying normalization. Figure 9 shows how the calendar time effects would have been estimated under alternative interpretation constraints. In order to assess the range of "reasonable" interpretation errors, we take the observed within-year differences across all ages between 33 and 42 as a starting point. We then assess the implications for our estimated time effects of assuming that the true effect of changing age from 37 to 38 (or from 42 to 43) rather than being zero - as we have assumed so far - is equal to the observed age differentials in this age interval. For example, the lines entitled "if true equals observed difference between age38-age37" show the estimated calendar effects if we substitute the observed withinyear difference between these two years for the assumed zero-difference. The other lines then show illustrate the impact of substituting the largest within-year observed differences (in both directions) between any ages in the age 33-42 interval. Our interpretation of the results is that the estimated calendar time effects are quite robust with respect to the selection of normalization.
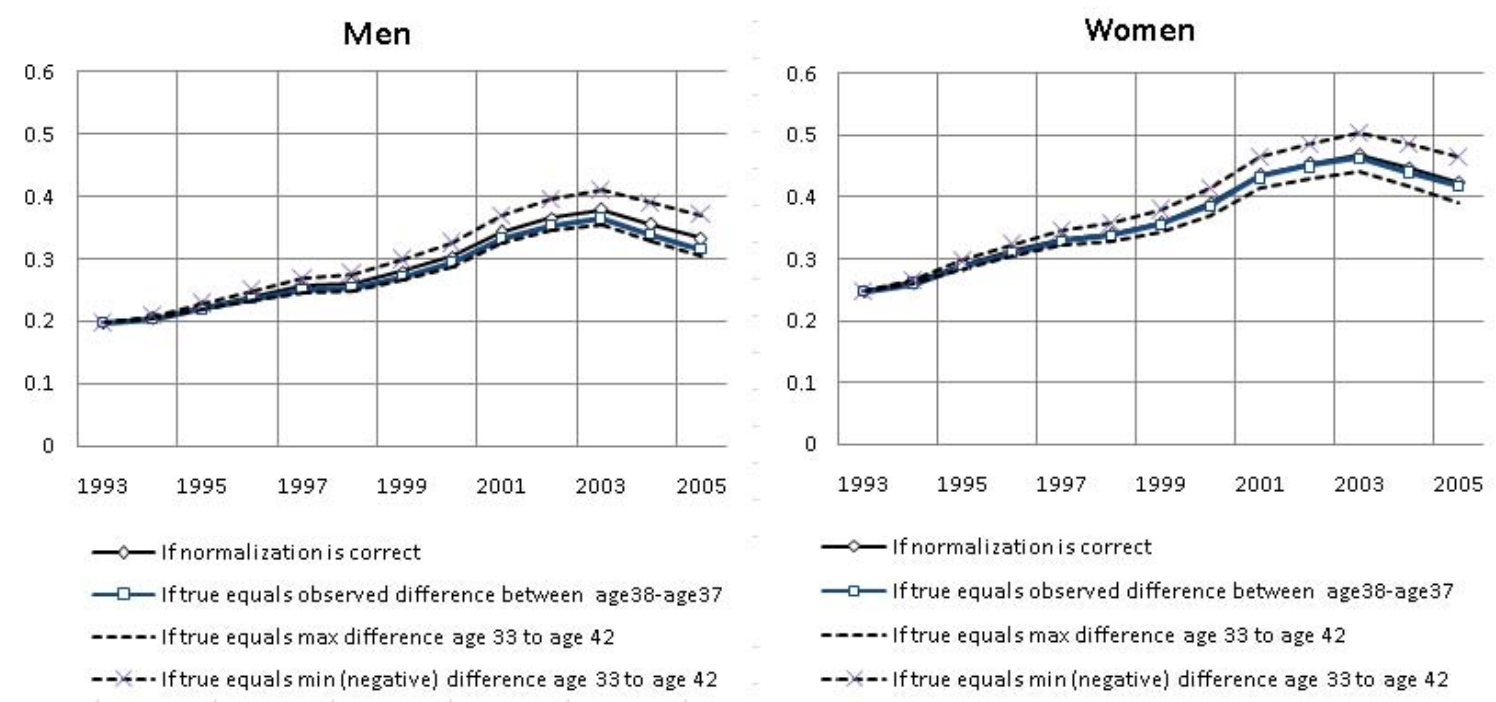

Figure 9. The estimated impacts of time on the probability of experiencing a long-tersickness episode under alternative interpretation constraints. 
Finally, we evaluate our findings' robustness with respect to the selection of outcome measure and statistical model. Figure 10 reports the estimated time-effects resulting from a fixed effect OLS model, with the number of absence days as the dependent variable, and with the same interpretation restriction as in the dichotomous model. ${ }^{6}$ The results from this model are clearly not directly comparable to the results from the dichotomous model, since the outcome measure is a different one. However, Figure 10 shows that the estimated calendar time profiles tell a similar story. Individual absence propensities have more than doubled from 1993 to 2005. Compared to the previously reported results (Figure 5) there is an interesting twist in the estimated effects for men, though, in that the within-individual changes over time no longer exceeds the observed aggregate changes.
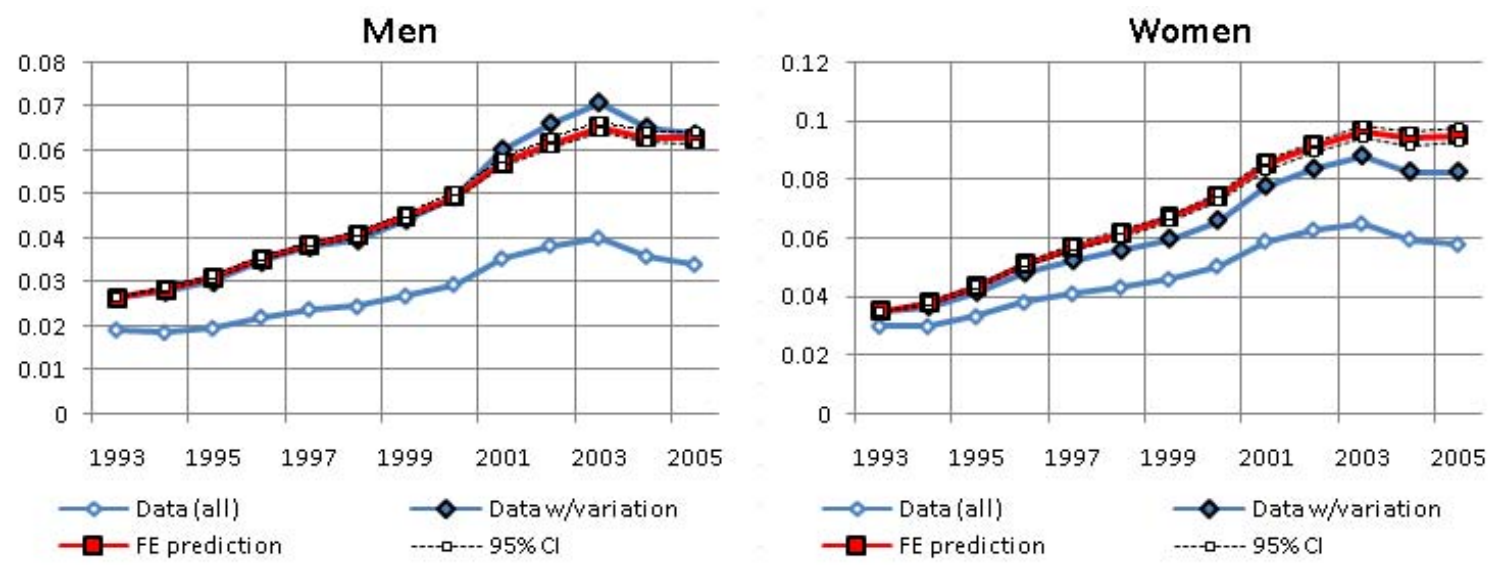

Figure 10. The estimated impacts of time on the on the annual number of registered absence days.

\section{Concluding remarks}

In this paper, we have examined the rise long-term in absenteeism from 1993 to 2005, with a focus on disentangling the roles of birth-cohort, age, and time. Our main finding is that the age-adjusted absence propensity has risen significantly within individuals, and

\footnotetext{
${ }^{6}$ The estimates reported here are from the conditional dataset (i.e., without last-year observations). Estimates based on data including last-year observations again produce very similar results.
} 
this within-individual rise is actually larger than the aggregate rise in absenteeism. While this finding does not allow us to identify a particular causal mechanism, it does make it possible to rule out some popular explanations. We can reject the popular hypothesis that the rise in absenteeism resulted from the entry of new cohorts into the labor market with weaker work-norms than older cohorts. We can also reject the potentially reassuring idea that it resulted from successful integration of marginal workers with poor health into the workforce. Our findings actually point to the contrary; i.e., that workers with high absence propensity have been systematically sorted out of the labor market. This finding is consistent with the fact that the fraction of the working-age population who were inactive due to permanent or temporary disability rose markedly in during our data period, from around 12 percent in 1993 to 16 percent in 2005. Our findings may thus indicate that the problem of rising absenteeism among the still-employed workers in Norway is even more worrying than indicated by aggregate statistics.

\section{References}

Askildsen, J. E., Bratberg, E. and Nilsen, Ø. A. (2005) Unemployment, Labour Force Composition and Sickness Absence. A Panel Data Study. Health Economics, Vol. 14, No. 11, 1087-1101.

Baltagi, B. H. (2008) Econometric Analysis of Panel Data, Fourth Edition, Chichester: Wiley

Biørn, E. (2010) Identifying Trend and Age Effects in Sickness Absence from Individual Data. Some Econometric Problems. Memorandum, Department of Economics, University of Oslo.

Cameron, A. C and Trivedi, P. K. (2005) Microeconometrics. Methods and Applications. Cambridge University Press, New York.

Chamberlain, G. (1984) Panel Data. In Handbook of Econometrics, Vol. 2, ed. by Z Gril- 
liches and M. D. Intriligator. Amsterdam: North Holland, 1247-1318.

Gans, D., and Silverstein, M. (2006) Norms of Filial Responsibility for Aging Parents Across Time and Generations. Journal of Marriage and Family, 68, 961-976.

Gaure, S. (2010) Dummy-encoding of Inherently Collinear Variables. Working Paper. In progress.

Greene, W. H. (2008) Econometric Analysis. Sixth Edition. New Jersey. Prentice Hall.

Hilbe, J. M. (2009) Logistic Regression Models. New York: Chapman \& Hall/CRC Press.

Ihlebaek, C., Brage, S., and Eriksen, H. R. (2007) Health Complaints and Sickness Absence in Norway, 1996-2003. Occupational Medicine, 57, 43-49.

Kupper, L. L., Janis, J. M., Salama, I. A., Yoshizawa, C. N. and Greenberg, B. G. (1983) Age-Period-Cohort Analysis: An Illustration of the Problems in Assessing Interaction in One Observation per Cell Data. Commun. Statist.-Theor. Meth. Vol. 12, No. 23, 2779-2807.

Lechner, M., Lollivier, S., and Magnac, T. (2008) Parametric Binary Choice Models. Chapter 7 in The Econometrics of Panel Data. Fundamentals and Recent Developments in Theory and Practice, Third Edition, Heidelberg: Springer.

Lindbeck, A. (1995) Hazardous Welfare-State Dynamics. American Economic Review, Papers and Proceedings, 85, 9-15.

Lindbeck, A., Nyberg, S., and Weibull, J. W. (1999) Social Norms and Economic Incentives in the Welfare State. Quarterly Journal of Economics, 114, 1-35.

Markussen, S. (2009a) Closing the Gates? Evidence from a Natural Experiment on Physicians' Sickness Certification. Memorandum No. 19/2009, Department of Economics, University of Oslo.

Markussen, S. (2009b) The Effects of Sick-Leaves on Earnings. Memorandum No. 20/2009, Department of Economics, University of Oslo. 
Markussen, S., Røed, K., Røgeberg, O. J., and Gaure, S. (2009) The Anatomy of Absenteeism. IZA Discussion Paper No. 4240.

Nordberg, M. and Røed, K. (2009) Economic Incentives, Business Cycles, and LongTerm Sickness Absence. Industrial Relations, Vol. 48, No. 2, 203-230.

Ryder, N. B. (1965) The Cohort as a Concept in the Study of Social Change. American Sociological Review, 30, 843-861.

\section{Appendix: The prediction distance measure $(\rho)$}

The prediction distance measure $(\rho)$, see Table 2, represents the distance between the predicted sequence based on the estimated model and the worst possible prediction that could have been made. We use the normalized Kendall distance, the number of adjacent exchange operations needed to transform one vector into another. We explain our measure by means of an example. Say we have 8 observations for an individual, 5 of them have positive outcome. Based on our estimated model, we sort the observations from lowest to the highest outcome probability. Our prediction is clearly that the five events occurred for the five observations with highest estimated outcome probability; i.e.

$$
00011111 \text {, }
$$

where 1 is a positive outcome and 0 is negative outcome. Now, say that the person in question actually had the following sequence of outcomes

$$
01111010 \text {. }
$$

Our prediction was clearly less than perfect in this case. But it could have been worse. The events could have happened for the five observations with the lowest estimated outcome probability, i.e.

11111000 .

How much better was our prediction than the worst possible prediction? We count the required moves: 


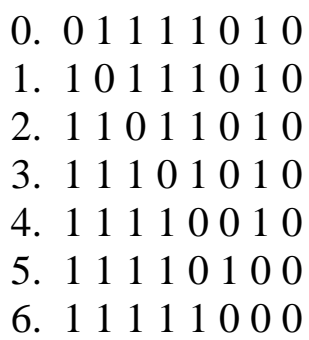

We then normalize by dividing by the distance between the best and worst prediction (which is 15 in this case). To avoid ambiguity, we provide a short R-snippet which takes as input a vector like the above, with 0 (or FALSE) for negative outcome, 1 (or TRUE) for positive outcome, and computes the distance.

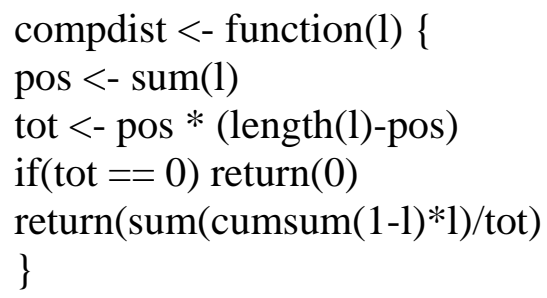

For individual $i$ we call this quantity $\rho_{i}$ and compute

$$
\rho=2\left(\frac{1}{N} \sum \rho_{i}-\frac{1}{2}\right)
$$

where $\mathrm{N}$ is the number of individuals with variation.

We have not fully investigated the properties of this $\rho$, but $\rho^{2}$ seems to have some sort of "percent of explained variation” interpretation. Moreover, for a given underlying data generation process, it seems to be independent of our observation length (we have tried this on artificial data with observation lengths between 2 and 60). $\rho$ is zero for random data, 1 for perfect prediction, and -1 for worst possible prediction. Changing the sign of all parameters used in the prediction merely changes the sign of $\rho$. In this distance context, the "correct year prediction” corresponds to using the Hamming distance as a distance measure, the "correct prediction sequence" corresponds to using the binary measure which is 1 for identical sequences, 0 otherwise. 